\title{
Ambiguity Results in the Characterization of $h v$-convex Polyominoes from Projections
}

Elena Barcucci ${ }^{1}$, Paolo Dulio ${ }^{2}$, Andrea Frosini ${ }^{1(凶)}$, and Simone Rinaldi ${ }^{3}$

1 Dipartimento di Matematica e Informatica "U. Dini”, Università di Firenze, viale Morgagni 65, 50134 Florence, Italy

\{elena.barcucci, andrea.frosini\}@unifi.it

2 Dipartimento di Matematica "F. Brioschi", Politecnico di Milano,

Piazza Leonardo da Vinci 32, 20133 Milan, Italy

paolo.dulio@polimi.it

3 Dipartimento di Ingegneria dell'Informazione e Scienze Matematiche, Università di Siena, Via Roma, 56, 53100 Siena, Italy

rinaldi@unisi.it

\begin{abstract}
In 1997 R. Gardner and P. Gritzmann proved a milestone result for uniqueness in Discrete Tomography: a finite convex discrete set can be uniquely determined by projections taken in any set of seven planar directions. The number of required directions can be reduced to 4 , providing their cross-ratio, arranged in order of increasing angle with the positive $\mathrm{x}$-axis, does not belong to the set $\{4 / 3,3 / 2,2,3,4\}$.

Later studies, supported by experimental evidence, allow us to conjecture that a similar result may also hold for the wider class of $h v$-convex polyominoes.

In this paper we shed some light on the differences between these two classes, providing new 4-tuples of discrete directions that do not lead to a unique reconstruction of $h v$-convex polyominoes. We reach our main result by a constructive process. This generates switching components along four directions by a recursive composition of only three of them, and then by shifting the obtained structure along the fourth one.

Furthermore, we stress the role that the horizontal and the vertical directions have in preserving the $h v$-convexity property. This is pointed out by showing that these often appear in the 4-tuples of directions that allow uniqueness.

A final characterization theorem for $h v$-convex polyominoes is still left as open question.
\end{abstract}

Keywords: Discrete geometry $\cdot$ Discrete tomography $\cdot h v$-convex set Uniqueness problem $\cdot$ Switching component

\section{Introduction}

We consider the problem of the characterization of finite discrete set of points of the $2 D$ integer lattice from projections, i.e., from the knowledge of the number of 
points lying on parallel lines along a given set of discrete directions. This research is of great relevance in Discrete Tomography, and it is motivated by the need of a faithful reconstruction of the internal structure of an object that is not directly accessible. The general problem has been studied since the beginning of the 50's, both from theoretical and computational perspectives (see [12] for a survey on the topic). Due to the existence of different sets of points that are consistent with the projections along any set of discrete directions (in [8], Fishburn et al. gave several characterizations of the finite subsets of $Z^{n}$ that are uniquely determined by their horizontal and vertical projections), constraints have been added to restrict the space of solutions to obtain faithfulness, say uniqueness, in the reconstruction process.

In [10], the authors provided the following milestone result that answers the uniqueness problem in case of convex polyominoes, i.e. the class $\mathcal{C}$ of connected sets of points that match their convex hulls:

Theorem 1. Let us consider the class $\mathcal{C}$; it holds that

1. if $U$ is a set of four discrete directions not having cross ratio $\{4 / 3,3 / 2,2,3,4\}$, then $\mathcal{C}$ is characterized by $U$ (i.e., uniquely determined by the projections along the directions of $U$ );

2. $\mathcal{C}$ is characterized by any set of seven mutually nonparallel directions;

3. there is a set of six discrete directions not characterizing $\mathcal{C}$;

4. $\mathcal{C}$ cannot be characterized by any set of three discrete directions.

For every set of four directions $U=\left\{u_{1}, u_{2}, u_{3}, u_{4}\right\}$, the cross ratio is defined as

$$
\rho(U)=\frac{\left(h_{3}-h_{1}\right)\left(h_{4}-h_{2}\right)}{\left(h_{3}-h_{2}\right)\left(h_{4}-h_{1}\right)},
$$

where $u_{i}=\left(u_{i x}, u_{i y}\right)$ and $h_{i}=\frac{u_{i y}}{u_{i x}}$, for $i=1,2,3,4$ and $0 \leq h_{1}<h_{2}<h_{3}<h_{4}$. If $u_{4}=(0,1)$, say by abuse of notation $h_{4}=\infty$, then we only keep the terms not containing $h_{4}$, and the cross ratio reduces to $\rho(U)=\frac{\left(h_{3}-h_{1}\right)}{\left(h_{3}-h_{2}\right)}$.

A major task in proving the above result relies on the analysis of the class of lattice $U$-polygons, consisting of any non-degenerate convex polygon $P$ such that, for any vertex $v$ of $P$, and for any direction $u \in U$, the line through $v$ and parallel to $u$ meets a different vertex $v^{\prime}$ of $P$. The proof of Theorem 1 comes out from a number of lemmas and properties that combine tools from $p$-adic valuations, projective geometry, algebraic number theory and convexity.

After the paper of Gardner and Gritzmann appeared, the leading idea was that, when a kind of convexity information is available, ambiguous reconstructions are somehow related to the existence of $U$-polygons. Therefore, understanding the properties of such structures can help in achieving uniqueness results and reconstruction hints (see [1-6] for interesting examples). In [4] it was proved that if $|U| \geq 4$, and the values of the cross ratio of any set of four directions in $U$, arranged in order of increasing angle with the positive $\mathrm{x}$-axis, are in the set $\{4 / 3,3 / 2,2,3,4\}$, then a lattice $U$-polygon does exist. All the lattice $U$-polygons exhibited in [4] are dodecagons, produced by tiling affinely regular hexagons. 
Such kind of hexagonal tiling has been investigated and generalized in [6,7], where several results concerning the symmetries of $U$-polygons, and their geometric structure have been also provided.

In [4], and later on in [9], some classes of polyominoes that are uniquely determined by projections are provided, combining the notion of horizontal and vertical convexity with additional geometrical constraints. The characterization of the class of $h v$-convex polyominoes, say $\mathcal{H V}$, from projections is still open: again in [4], it was conjectured that Theorem 1 continues to hold for the elements of this class.

Our study sheds some new light on this problem: we restrict the conjecture by showing that ambiguities in the reconstruction do persist for any set of directions whose elements belong to the same quadrant and do not include both the horizontal and the vertical ones. Then, we go further by showing that no characterization is possible for any set of four directions including the two axial ones and such that the remaining two form a sufficiently small angle.

The overall organization of the paper includes, in the next section, a review to the basics of discrete tomography and the related uniqueness problem. Then, Sect. 3 presents our main results, i.e., the construction of specific $h v$-switchings along a class of 4 -tuples of directions. The prominent role of the projections along the horizontal and vertical directions is also underlined. Finally, in Sect. 4 we provide perspectives and open problems related to the definition of $h v$-convex polyominoes from a generic set of given projections.

\section{Definitions and Known Results}

A finite discrete subset of points $S$ in the integer lattice is usually represented by a set of cells (unit squares whose centers are the points in the set itself) on a squared surface, and its dimensions are those of the minimal bounding rectangle. We choose to identify the lower leftmost cell of such rectangle with the origin of the integer lattice, so that each set of points will be considered up to translation.

A polyomino is a finite union of cells whose interior is connected (see Fig. 1(a)). A column (row) of a polyomino is the intersection between the polyomino and an infinite strip of cells whose centers lie on a vertical (horizontal) line.

Nevertheless, several subclasses of interest were considered by putting on polyominoes constraints defined by the notion of convexity along different directions. In particular, a polyomino is $h$-convex (resp. $v$-convex) if each of its rows (resp. columns) is connected. A polyomino is $h v$-convex, if it is both $h$-convex and $v$-convex (see Fig. 1(b)). Finally, a polyomino is convex if it is convex w.r.t. all the discrete directions, i.e., if it equals its discrete convex hull (see Fig. 1(c)).

Let $u=\left(u_{x}, u_{y}\right)$ be a discrete direction i.e., a couple of coprime integers. To each discrete set $S$ and direction $u$, we associate an integer vector $X_{u}(S)$ that stores the number of points of $S$ that lie on each line parallel to $u$ and intersecting the minimal bounding rectangle of $S$; we indicate such a vector as the (vector of) projections of $S$ along $u$ (see Fig. 1(c)). 


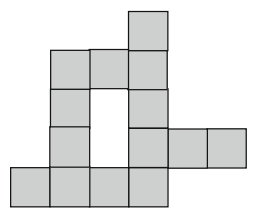

(a)

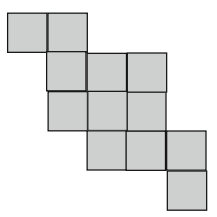

(b)

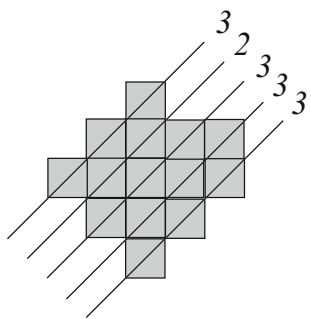

(c)

Fig. 1. (a) a polyomino; (b) an $h v$-convex polyomino; (c) a convex polyomino and its projections along the direction $u=(1,1)$.

Let $U$ be a finite set of discrete directions. We say that $S$ is determined by (the projections along the directions of) $U$, if $X_{u}(R)=X_{u}(S)$ implies $R=S$. We say that $S$ is ambiguous w.r.t. $U$ otherwise. Finally, a class of discrete sets $\mathcal{S}$ is characterized by $U$ if all its elements are non ambiguous w.r.t. $U$ inside the class.

One of the main aims in the field of Discrete Tomography is the achievement of a faithful reconstruction of an unknown object, regarded as a discrete set of points at a certain resolution, from a set of projections. As one can imagine, the existence of different sets of points sharing the same set of projections may dramatically change into meaningless the whole process, so the relevance of the following problem:

\section{Uniqueness $(\mathcal{S}, U)$}

Instance: a class of discrete sets $\mathcal{S}$, and a set of discrete directions $U$.

Question: Does there exist two distinct elements of $\mathcal{S}$ having the same projections along the directions of $U$ ?

\subsection{The Notion of Switching Components}

Many authors studied Uniqueness $(\mathcal{S}, U)$ in terms of the cardinality and the characteristics of the class of discrete sets sharing the same projections along $U$ and, since the very beginning, they relied on the idea of switching component (in a very first study, Ryser [14] called it interchange) i.e., a rearranging of some elements of a set that preserves the projections along $U$.

More precisely, a discrete set $S$ contains a switching component along $U$ if there is a set of cells $S^{\prime}$ in the corresponding minimal bounding rectangle such that:

- $S^{\prime}=S^{0} \cup S^{1}, S^{0} \cap S^{1}=\emptyset$ and $\left|S^{0}\right|=\left|S^{1}\right|$;

- if $a \in S^{0}$ and $b \in S^{1}$, then exactly one among $a$ and $b$ belongs to $S$;

- for each $u \in U, X_{u}\left(S^{0}\right)=X_{u}\left(S^{1}\right)$ i.e., each line parallel to one direction of $U$ contains the same number of elements in $S^{0}$ and $S^{1}$.

We call $S^{\prime}$ a switching component along $U$. 


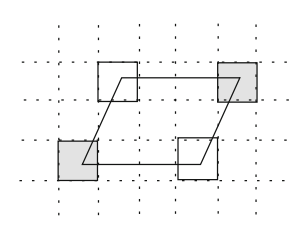

(a)

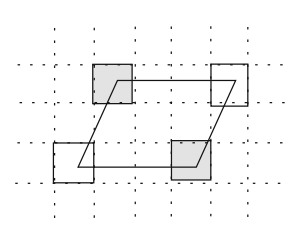

(b)

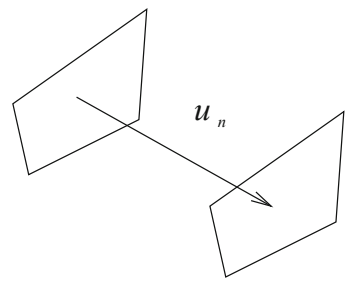

(c)

Fig. 2. (a) the switching components $S^{\prime}$ and its two sets $S^{0}$ and $S^{1}$ along the directions $(1,0)$ and $(1,2)$. The dark squares are elements of $S ;(b)$ the switching component $\bar{S}^{\prime}$ obtained by $S^{\prime}$ after changing the values of its elements; $(c)$ the recursive step of the construction of a switching along the $n$-th direction $u_{n}$.

Figure 2(a), shows the two different switching components $S^{0}$ and $S^{1}$ along two directions, (the light gray cells are not elements of $S$ ).

From the definition of switching component, it holds that if a set $S$ has a switching component $S^{\prime}$ along $U$, then by changing the values of the elements of $S^{\prime}$, we obtain another set having the same projections. We denote this discrete set by $\hat{S}$. Furthermore, we call the switching component obtained by changing the values of all the elements of $S^{\prime}$, its dual, and we indicate it by $\bar{S}^{\prime}$ (see Fig. $2(b))$. Obviously the dual operator has period 2. We recall the following result (see [13]):

Theorem 2. Let $\mathcal{S}$ be a class of discrete sets. An element $S \in \mathcal{S}$ is ambiguous (in $\mathcal{S}$ ) w.r.t. to a set of directions $U$, if and only if $S$ has a switching component along $U$ such that $\hat{S} \in \mathcal{S}$.

The following simple property, rediscovered several times (see [12]), introduces a recursive way to generate switching components along a generic set of discrete directions:

Property 1. Let $S^{\prime}$ be a switching component along $u_{1}, u_{2}, \ldots, u_{n-1}$, and $S^{\prime \prime}$ be the dual of the translation of $S^{\prime}$ along the further direction $u_{n}$ so that $S^{\prime} \cap S^{\prime \prime}=\emptyset$, i.e., $S^{\prime \prime}=\left\{k u_{n}+(i, j):(i, j) \in \bar{S}^{\prime}\right\}$, for $k$ sufficiently large integer. The set $S^{\prime} \cup S^{\prime \prime}$ is a switching component along the directions $u_{1}, u_{2}, \ldots, u_{n}$.

From this property, two main consequences follow:

(i) we can construct a switching component along a generic set $U$ of directions in the recursive way shown in Fig. 2;

(ii) since we can construct a switching component along every $U$, then the class of all the possible discrete sets cannot be characterized by any set of discrete directions. 

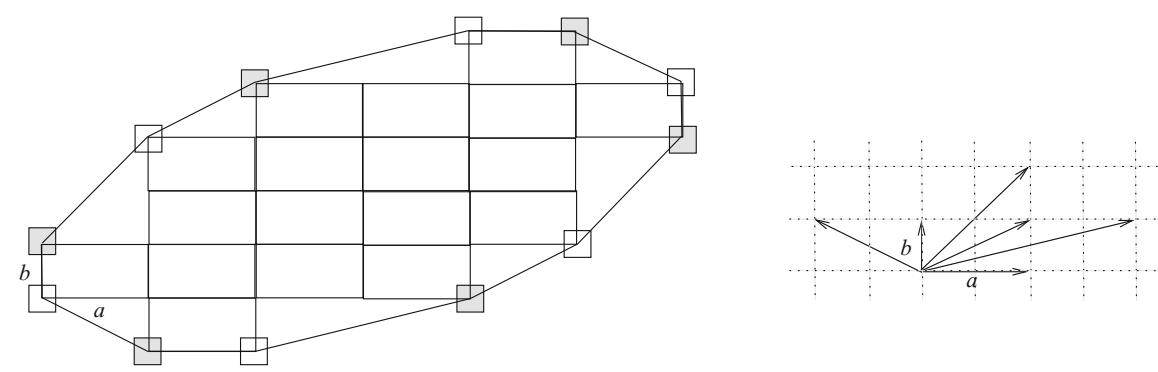

Fig. 3. An example of a (dodecagonal) switching component along the directions $(1,0)$, $(0,1),(a, b),(-a, b),(a, 2 b)$, and $(2 a, b)$.

We stress that the switching components may also be composed in order to achieve complex switching configurations that connect all the elements sharing the same projections to form a connected graph structure.

If we restrict our study to the class $\mathcal{C}$ of convex polyominoes, Theorem 1 states necessary and sufficient conditions in order for a discrete set to be characterized by projections. In particular, any set of seven directions provides uniqueness, while six do not always suffice as the $U$-dodecagon in Fig. 3 shows, since every set of four directions in $U$ returns a value of the cross ratio belonging to $\{4 / 3,3 / 2,2,3,4\}$.

On the other hand, if we relax too much the constraints on the convexity of the sets, for example preserving convexity only along the horizontal direction, then a negative result holds, as shown in [4], Theorem 3.7:

Theorem 3. No finite set of directions characterizes the class of horizontally (resp. vertically) convex polyominoes.

Our intent is to inspect the cut-off line between those results that seems to show up when both the horizontal and vertical convexity are present.

In the same paper, it has been proposed the following

Conjecture 1. The class $\mathcal{H} \mathcal{V}$ is characterized by a set of four discrete directions $U=\left\{(1,0),(0,1), u_{3}, u_{4}\right\}$ such that $\rho(U)$ does not belong to $\{4 / 3,3 / 2,2,3,4\}$.

that has been supported by computational evidence: $h v$-convex polyominoes were randomly generated and each of them reconstructed using its projections along a set of four directions whose cross ratio does not belong to $\{4 / 3,3 / 2,2,3,4\}$. It was verified that the algorithm uniquely reconstructed the generated $h v$-convex polyominoes. In the next section, we modify the conjecture by showing a new class of 4-tuples of directions that allow switching components in $h v$-convex polyominoes.

\section{A New Class of Switching Components}

The following result from $[4,11]$, provides a step forward to the study of the uniqueness problem on the class $\mathcal{H} \mathcal{V}$ : 

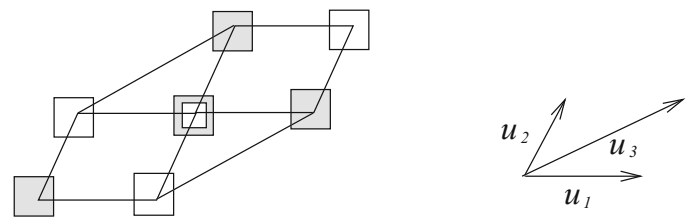

Fig. 4. The construction of the hexagonal switching along three directions $u_{1}, u_{2}$, and $u_{3}$.

Property 2. Let $u_{1}, u_{2}$, and $u_{3}$ be three discrete directions. There is a hexagonal switching $H$ along $U=\left\{u_{1}, u_{2}, u_{3}\right\}$.

Proof. We prove the statement by construction. First of all we construct the switching $S$ along $u_{1}$ and $u_{2}$, following Property 1. Then, we extend both the sides of $S$ until they equal a $k$ multiple of $u_{3}$, and finally we add to $S$ its dual along $k u_{3}$, i.e. $H=S \cup k u_{3}+\bar{S}$. The two coincident points are deleted (see Fig. 4).

Observe that hexagonal switching components have just six distinct points, which is the minimal cardinality for a three directions' switching, while the general construction along three directions defined in Property 1 requires eight points. Furthermore, each hexagonal switching is convex in the sense that it is a switching of a convex set.

However, from Property 2 it is easy to realize that hexagonal switching components are not different from those defined in Property 1: as a matter of fact, they can be obtained by translating along the direction $u_{3}$ an appropriate magnification of the switching components along $u_{1}$ and $u_{2}$, so that two opposite of the eight points coincide and so annihilate.

Let $U=\left\{u_{1}, u_{2}, u_{3}\right\}$ be a set of three directions in $\mathbb{R}^{2}$. The group $\mathcal{S}_{3}^{U}$ which permutes the indices $\{1,2,3\}$ can be represented as a group of symmetries fixing a triangle $T$ with edges parallel to $\left\{u_{1}, u_{2}, u_{3}\right\}$. In [7, Theorem 6], the following characterization of lattice $U$-polygons has been determined

Theorem 4. Let $U=\left\{u_{1}, u_{2}, u_{3}\right\}$ be any set of three lattice directions. Let $P$ be a lattice hexagon. Then $P$ is a $U$-polygon if and only if $\mathcal{S}_{3}^{U}(P)=P$.

As a consequence, a hexagonal switching along $U=\left\{u_{1}, u_{2}, u_{3}\right\}$ always returns a rational magnification of a lattice $U$-hexagon $P$, where the triangle $T$ reduces to its barycenter.

Property 3. The class $\mathcal{H} \mathcal{V}$ cannot be characterized by a finite set of discrete directions $U$ such that:

(i) at most one among the directions $h=(1,0)$, and $v=(0,1)$ belongs to $U$; and

(ii) all the directions in $U$ belong to the same quadrant. 
This property predicts the basic role played by the two convexity directions $h$ and $v$ when they belong to $U$; for brevity we omit its proof, that is related to the possibility of connecting sufficiently distant parts of a switching component defined as in Property 1 with a path that preserves $h v$-convexity. It is also easy to verify that the same construction holds when the axes direction is replaced by a generic one:

Property 4. The class $\mathcal{H} \mathcal{V}$ cannot be characterized by a finite set of discrete directions $U$ not containing $(1,0)$ and $(0,1)$ and such that all but one lie in the same quadrant.

Relying on these results, we focus on the characterization of $\mathcal{H V}$ by means of a set of four directions $U=\left\{h, v, u_{3}, u_{4}\right\}$ such that $\rho(U) \notin\{4 / 3,3 / 2,2,3,4\}$.

\subsection{Composing Hexagonal Switching Components Along a Diagonal Direction}

The definition of hexagonal switching component along three directions suggests a possible construction of $h v$-convex switching operations along four directions. From [7, Corollary 7] we know that an hexagon $H$ is a lattice $U$-polygon if and only if for any two diagonals of $H$, having direction $v_{1}, v_{2}$, there exists a symme$\operatorname{try} \sigma \in \mathcal{S}_{3}^{U}$ such that $\sigma\left(\left\{u_{1}, u_{2}, u_{3}\right\}\right)=\left\{u_{1}, u_{2}, u_{3}\right\}$, and $\sigma\left(v_{1}\right)=v_{2}$. This induces to explore further switchings of a hexagonal one when performed along a diagonal direction. We define such switchings as diagonal-hexagon switchings. Also, up to affine transformation, we can always assume that $U=\{(a, 0),(0, b),(a, b)\}$.

Property 5. For any three integer numbers $a, b, k$

(i) there exists an $h v$-convex hexagonal switching $H_{k}$ along the set of three directions $U=\{(a, 0),(0, b),(a, b)\}$;

(ii) there exists an $h v$-convex diagonal-hexagon switching $H_{k}^{\prime}$ along $U_{k}^{\prime}=U \cup$ $\{(2 a, 2 b)+k(2 a, b)\}$. The same holds if $U_{k}^{\prime}=U \cup\{(2 a, 2 b)+k(a, 2 b)\}$.

Proof. Let us consider the set $U=\{(a, 0),(0, b),(a, b)\}$ and construct the related hexagonal switching $H$. Then, we translate it along the direction $(2 a, b)$, and we annihilate the incident points having opposite values. The obtained ten points configuration, say $H_{1}=H \cup((2 a, b)+H)$, is still an $h v$-convex switching along $U$. Now we define $H_{1}^{\prime}=H_{1} \cup\left((4 a, 3 b)+\bar{H}_{1}\right)$. It is immediate to see that $H_{1}^{\prime}$ is an $h v$ convex switching along $U^{\prime}=U \cup\{(4 a, 3 b)\}$. Now, by iterating the construction for $k$ times, we get

$$
H_{k}=H \bigcup_{i=1}^{k}(k(2 a, b)+H) \quad \text { and } \quad H_{k}^{\prime}=H_{k} \cup\left((2 a, 2 b)+k(2 a, b)+\bar{H}_{k}\right) .
$$

Then $H_{k}^{\prime}$ provides an $h v$-convex switching component with respect to the set $U_{k}^{\prime}=U \cup\{((2 a, 2 b)+k(2 a, b))\}$. In the case $U_{k}^{\prime}=U \cup\{((2 a, 2 b)+k(a, 2 b))\}$, the statement follows with a quite analogous argument. 
Theorem 5. The cross ratio of any diagonal-hexagon switching $U_{k}^{\prime}$ is

$$
\rho\left(U_{k}^{\prime}\right)=2 \frac{k+1}{k} .
$$

Proof. Suppose that $U_{k}^{\prime}=U \cup\{((2 a, 2 b)+k(2 a, b))\}$. Then, being $a, b, k>0$, the slope $m_{k}$ of the $k$-dependent direction of $U_{k}^{\prime}$ satisfies

$$
0<m_{k}=\frac{(k+2) b}{2(k+1) a}<\frac{b}{a} .
$$

Assume the slope as a projective coordinate. Then, by arranging increasingly the four slopes of the directions in $U_{k}^{\prime}$, the computation of their cross ratio $\rho\left(U_{k}^{\prime}\right)$ provides

$$
\rho\left(U_{k}^{\prime}\right)=\left(0, m_{k}, \frac{b}{a}, \infty\right)=2 \frac{k+1}{k} .
$$

In case $U_{k}^{\prime}=U \cup\{((2 a, 2 b)+k(a, 2 b))\}$, it results

$$
0<\frac{b}{a}<m_{4}=\frac{2(k+1) b}{(k+2) a},
$$

so that

$$
\rho\left(U_{k}^{\prime}\right)=\left(0, \frac{b}{a}, m_{k}, \infty\right)=2 \frac{k+1}{k},
$$

and the statement follows.

Remark 1. Note that $\rho\left(U_{1}^{\prime}\right)=4, \rho\left(U_{2}^{\prime}\right)=3$, and

$$
\lim _{k \rightarrow \infty} \rho\left(U_{k}^{\prime}\right)=2 .
$$

Moreover, up to rearranging the order of the directions, the values $4 / 3$ and $3 / 2$ can be turned to 4 and 3 , respectively. This points out that the set of cross ratio determined by diagonal-hexagon switching

$$
\left\{2 \frac{k+1}{k}, k \in \mathbb{N},\right\},
$$

naturally refines the set $\{4 / 3,3 / 2,2,3,4\}$, that prevents uniqueness in the class $\mathcal{C}$ of convex lattice sets.

Figure 5 shows the above result in the case when $k=3$ and the related switching along the directions $U^{\prime}=\{(a, 0),(0, b),(a, b),(8 a, 5 b)\}$ whose cross ratio is $8 / 3$. 


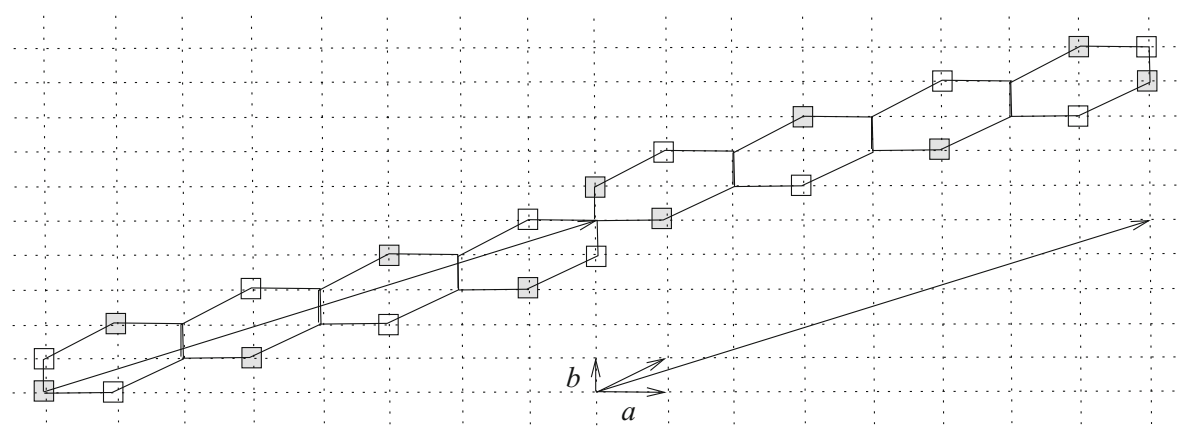

Fig. 5. The composition of four hexagonal switchings, i.e., $k=3$ w.r.t. the set $U=$ $\{(a, 0),(b, 0),(a, b)\}$ along the direction $(2 a, b)$ and the related switching components. The fourth obtained direction is $(8 a, 5 b)$.

\subsection{General Construction of the Switching Components}

Finally, we push to the last step our construction of a new class of $h v$-convex switching components by observing that successive shifts along one of the two directions $u=(2 a, b)$ or $v=(a, 2 b)$ of the hexagon $H$ can also generate new 4-tuples of elements for the set of directions $U^{\prime}$.

In particular, let us consider a sequence of $k_{1}$ successive shifts of $H$ along $u$ and $k_{2}$ along $v$ : the obtained switching along $U$, say $H_{k_{1}, k_{2}}$ is represented in Fig. 6(a). Following Eq. (1), we define

$$
H_{k_{1}, k_{2}}^{\prime}=H_{k_{1}, k_{2}} \cup\left((2 a, 2 b)+k_{1}(2 a, b)+k_{2}(a, 2 b)+\bar{H}_{k_{1}, k_{2}}\right) .
$$

The following holds

Theorem 6. The set $H_{k_{1}, k_{2}}^{\prime}$ is an hv-convex switching along the directions $U^{\prime}=$ $U \cup\left\{u_{4}\right\}$, with $u_{4}=(2 a, 2 b)+k_{1} u+k_{2} v$.

The proof can be achieved after observing that $H_{k_{1}, k_{2}}$ is an $h v$-convex switching along $U$, and the union with its dual, once translated along the direction $u_{4}$, preserve both the projections along $u_{4}$, by definition, and the $h v$-convexity.

Figure $6(b)$ shows an example of the switching when $k_{1}=1$ and $k_{2}=3$. We again underline that the cross ratio of the obtained direction $u_{4}=\left(2 a+k_{1} 2 a+\right.$ $\left.k_{2}, 2 b+k_{1} b+k_{2} 2 b\right)=(7 a, 9 b)$ does not belong to the set $\{4 / 3,3 / 2,2,3,4\}$.

After defining the set of discrete directions

$$
D=\left\{\left(2 a+k_{1} 2 a+k_{2}, 2 b+k_{1} b+k_{2} 2 b\right): k_{1}, k_{2} \in \mathbb{N}\right\},
$$

Theorem 6 allows us to modify Conjecture 1 as follows:

Conjecture 2. The class of $h v$-convex polyominoes is characterized by a set of four discrete directions $U=\left\{(1,0),(0,1),(a, b), u_{4}\right\}$ such that:

- $\rho(U) \notin\{4 / 3,3 / 2,2,3,4\}$;

- $u_{4} \notin D$. 


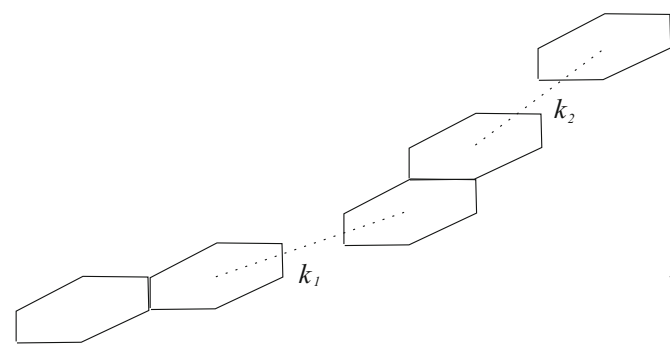

(a)

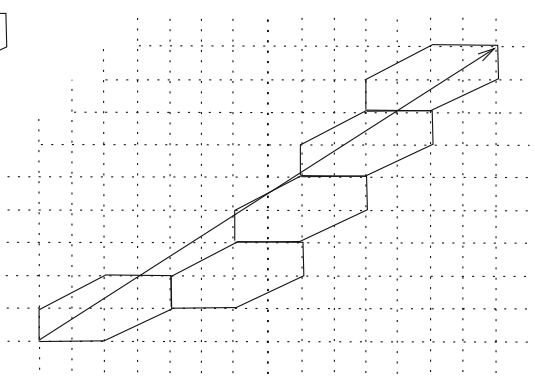

(b)

Fig. 6. $(a)$ a generic composition of $k_{1}$ hexagonal switchings along the direction $(2 a, b)$ and $k_{2}$ along the direction $(a, 2 b) ;(b)$ an example when $k_{1}=1$ and $k_{2}=3$. The obtained fourth direction is $(7 a, 9 b)$.

\section{Conclusions and Perspectives}

In this paper we have addressed the problem of generalizing the results of [10], related to the lattice convex sets, to the wider class $\mathcal{H} \mathcal{V}$ of $h v$-convex polyominoes. We have obtained a few preliminary properties towards a detailed answer to Conjecture 1. In particular we have obtained a complete characterization of diagonal-hexagon switching in $\mathcal{H} \mathcal{V}$, and we have determined all the possible values of the cross ratio involved in the corresponding ambiguous reconstructions. These have been explicitly exhibited also from the geometric point of view.

As a further step we wish to investigate different extensions of the presented constructions, in order to get a general description of all switchings preserving $h v$-convexity. This would allow to get a uniqueness result for $h v$-convex polyominoes, as well as to get new information on the geometric meaning of the values of the cross ratio related to ambiguous reconstructions

Acknowledgment. This study has been partially supported by INDAM - GNCS Project 2016.

\section{References}

1. Alpers, A., Larman, D.G.: The smallest sets of points not determined by their X-rays. Bull. London Math. Soc. 47, 171-176 (2015)

2. Alpers, A., Tijdeman, R.: The two-dimensional Prouhet-Tarry-Escott problem. J. Number Theor. 123, 403-412 (2007)

3. Brunetti, S., Dulio, P., Hajdu, L., Peri, C.: Ghosts in discrete tomography. J. Math. Imaging Vis. 53(2), 210-224 (2015)

4. Barcucci, E., Del Lungo, A., Nivat, M., Pinzani, R.: X-rays characterizing some classes of discrete sets. Linear Algebra Appl. 339, 3-21 (2001)

5. Cipolla, M., Lo Bosco, G., Millonzi, F., Valenti, C.: An island strategy for memetic discrete tomography reconstruction. Inform. Sci. 257, 357-368 (2104) 
6. Dulio, P.: Convex decomposition of $U$-polygons. Theor. Comput. Sci. 406, 80-89 (2008)

7. Dulio, P., Peri, C.: On the geometric structure of lattice U-polygons. Discrete Math. 307, 2330-2340 (2007)

8. Fishburn, P., Lagarias, J., Reeds, J., Shepp, L.: Sets uniquely determined by projections on axes II. Discrete Appl. Math. 91, 149-159 (1991)

9. Castiglione, G., Frosini, A., Restivo, A., Rinaldi, S.: Enumeration of L-convex polyominoes by rows and columns. Theor. Comput. Sci. 347(1-2), 336-352 (2005)

10. Gardner, R.J., Gritzmann, P.: Discrete tomography: determination of finite sets by X-rays. Trans. Amer. Math. Soc. 349, 2271-2295 (1997)

11. Gardner, R., Gritzmann, P.: Uniqueness and complexity in discrete tomography. In: Herman, G., Kuba, A. (eds.) Discrete Tomography: Foundations, Algorithms, and Applications, pp. 85-113. Birkhäuser, Basel (1999)

12. Herman, G.T., Kuba, A. (eds.): Discrete tomography: Foundations algorithms and applications. Birkhauser, Boston (1999)

13. Kuba, A.: Reconstruction of unique binary matrices with prescribed elements. Acta Cybern. 12, 57-70 (1995)

14. Ryser, H.: Combinatorial Mathematics, The Carus Mathematical Monographs No. 14, The Mathematical Association of America, Rahway (1963) 